\title{
Replacement of Coarse Aggregate and Fine Aggregate by Polyethylene Terephthalate in Light Weight Concrete
}

\author{
Swati Dhiman* and Harvinder Singh \\ Civil Engineering Department, Chandigarh University, Mohali - 140413, Punjab, India; \\ swatidhimansoni@gmail.com, Harvindersingh3630@gmail.com
}

\begin{abstract}
Objective: To find the property of a concrete mixture where conventional coarse and fine aggregate has been replace with PET granules at various ratios and test its suitability in real word scenario by various testing methods. Method: Test sample 108 in numbers were made and were tested at duration of 7 and 28 days. Also these samples had different replacement level so that optimum replacement ratio could be obtained. Various testing methods like compressive test, split tensile test and flexural test were performed to obtain statistical properties and characteristics of the concrete. Finding: It has been found that at replacement ratio of $10 \%$, the compressive strength obtained was maximum. The optimum ratio for flexural test and split tensile test is also $10 \%$. At this ratio the property of PET concrete is better than or equal to conventional concrete. Application: Around 335 million tons of plastic is produce annually worldwide of which $85 \%$ is not recycled. It can be used in concrete making to solve the problem of pollution and can help in saving natural resource. Also further study can focus on usability of light weight concrete in loose soil and in earthquake zone.
\end{abstract}

Keywords: Coarse Aggregate, Fine Aggregate, Light Weight Concrete, PET (PolyEthylene Terephthalate)

\section{Introduction}

\subsection{Background}

The Polyethylene Terephthalate (PET) for food or beverage packing is already familiar in public. After the contents are consumed, the PET plastic containers usually are directly discharged ${ }^{1}$. In present phase of industrialization, Plastic ought to be universally present artificial thing to be ever made which also has undue ramification as critical environmental hazard. Previous researchers revealed that $\mathrm{PET}$ waste fiber in concrete has significant role in terms of bonding and strength. PET waste has very weak bond with cement paste. Recycling and reusing are presently the only viable solution to this epic waste creation. PET and polypropylene have a huge chunk of about $20 \%$ and $18 \%$ respectively ${ }^{2,3}$. Globally, production of plastic-type and associated waste in 1950 stood at 1.7 Mt. Come 2012, it has multiplied by approx. 150 times and is $288 \mathrm{Mt}^{4}$. while rest of the polymericfamily waste stands at $37 \%$. Although huge amount of plastic waste goes to recycling but majority of it lands up as land/marine waste ${ }^{5}$. Plastic has inherent property of low-density which makes it viable option to be used as light substitutes. Traditional concrete is known to be water intensive and prone to shrinking shortcoming ${ }^{6}$. Concrete of the Arabian zone is known to exhibit similar trend $\frac{7,8}{.}$. Recycle PET fiber have proved to be effective in strength improvement at $0.7 \%$ of total weight of asphalt concrete mixture $\stackrel{9,10}{ }$.

PET fibers show better application and have properties unique compared to ordinary fibers like:

i) Chemical inertness.

ii) Corrosion resistant.

iii) Easier concrete setting. 
iv) They are lighter weight vis-a-vis steel fibers.

v) Improved regulation of cracking and shrinkage in plastic.

Hence, reusing of PET wastes in the building industry is an effective approach in both, preventing environmental pollution and designing economical buildings.

\subsection{Study Objectives}

Advantage of present study are to make the light weight concrete use of PET aggregate and give the more strength for the building and utilize the waste plastic. This study also for the future researches who work on this topic and inspire to create the other light concrete for new structure.

\subsection{Problem Limitations}

This study is obtaining the specification of 7 days and 28 days concrete with mix design calculation based on IS 10262-2009. This study is for M30 grade and the targeted strength is $30 \mathrm{MPa}$.

Table 1. Total number of trials

\begin{tabular}{|c|c|c|c|c|c|c|c|}
\hline & \multirow{2}{*}{$\begin{array}{c}\text { percentage of } \\
\text { PET(\%) }\end{array}$} & & 7 Days & & \multicolumn{4}{c|}{28 days } \\
\cline { 3 - 8 } & 0 & 3 & 3 & 3 & 3 & 3 & 3 \\
\hline conventional & 10 & 3 & 3 & 3 & 3 & 3 & 3 \\
\hline trial 1 & 20 & 3 & 3 & 3 & 3 & 3 & 3 \\
\hline trial 2 & 30 & 3 & 3 & 3 & 3 & 3 & 3 \\
\hline trial 3 & 35 & 3 & 3 & 3 & 3 & 3 & 3 \\
\hline trial 4 & 40 & 3 & 3 & 3 & 3 & 3 & 3 \\
\hline trial 5 & & 18 & 18 & 18 & 18 & 18 & 18 \\
\hline Total & & & 108 & & & & 3 \\
\hline Total of all & & & & & 3 & 3 \\
\hline
\end{tabular}

The casting (using varying amount of PET) was carried out and eighteen trials where done on cubes of dimension 150 cubic mm, dimension of cylinder $150 \mathrm{~mm}$ diameter and $300 \mathrm{~mm}$ height and beam size $100 \mathrm{~mm} \times$ $100 \mathrm{~mm} \times 500 \mathrm{~mm}$ and than compression was made with conventional concrete. After a time period of seven and twenty eight days specimen where subjected to split tensile test, flexural test and compression test. As we can see Table 1 various trials have been done with different percentage of PET in composition.

The purchased PET aggregation has been shown in Figure 1.

\section{Methodology}

The PET plastic waste used in this project in aggregate size was purchased from 'Royal Plastic Manufacturer' shop in Chandigarh where all types of plastic bottles and plastic ropes are manufactured. The size of plastic waste PET used in this project work is $4 \mathrm{~mm}$ to $10 \mathrm{~mm}$ to replace the coarse aggregate and fine aggregate. The code for mix design used is IS 10262-2009.

\subsection{Materials}

Cement used : Ordinary Portland - 43 grades.

Fine aggregate : Sand.

Coarse aggregate : $18 \mathrm{~mm}-60 \%$ and $10 \mathrm{~mm}-40 \%$.

Plastic fibers : PET aggregate $4 \mathrm{~mm}-10 \mathrm{~mm}$.

\subsection{Experimental Plan}

In this project concrete grade is M30 and the percent of replaced fine and coarse aggregate are $10 \%, 20 \%, 30 \%, 35 \%$, and $40 \%$. Fine and coarse aggregate replacement stands at $10 \%$ i.e., $5 \%$ of fine aggregate and $5 \%$ of coarse aggregate. As all percentage like be this type. 


\subsection{Mix Design}

M30 grade concrete numerical figures have been obtained using IS 456:2000 while IS 10262:2009 for the mix design. The materials used in this study have been mentioned in Table 2.

Table 2. Materials required as per is method of design

\begin{tabular}{|c|c|c|c|}
\hline $\begin{array}{r}\mathrm{W} / \mathrm{c} \\
\text { Ratio }\end{array}$ & $\begin{array}{c}\text { Cement } \\
\left(\mathrm{kg} / \mathrm{m}^{3}\right)\end{array}$ & $\begin{array}{c}\text { Fine aggregate } \\
\left(\mathrm{kg} / \mathrm{m}^{3}\right)\end{array}$ & $\begin{array}{c}\text { Coarse aggregate } \\
\left(\mathrm{kg} / \mathrm{m}^{3}\right)\end{array}$ \\
\hline 0.45 & 425.73 & 672.72 & 1187.87 \\
\hline
\end{tabular}

The properties of materials used are:

S.G of cement $=3.15$

S.G of fine aggregate $=2.70$

S.G of coarse aggregate $=2.80$

S.G of PET aggregate $=0.904$

Water absorption capacity

Coarse aggregate $=0.5 \%$

Fine aggregate $=1 \%$

The mix design picture has been shown in Figure 2.

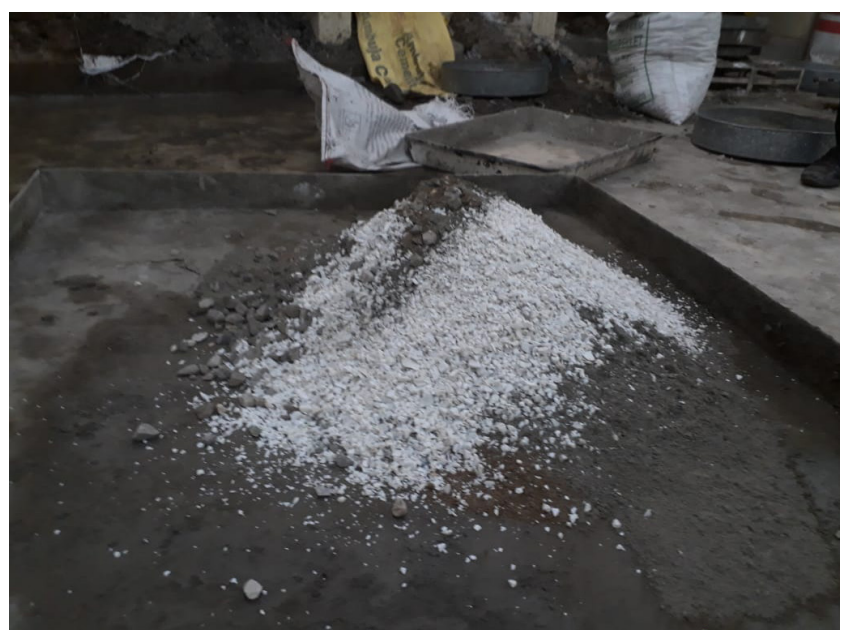

Figure 2. Dry mixed materials.

The graphical representation in Figure 4 depicts compressive strength of sample cube with varying ratio of coarse aggregate and fine aggregate by PET.

Figure 4 shows the testing results of compressive strength in different percentages of replacement. In the

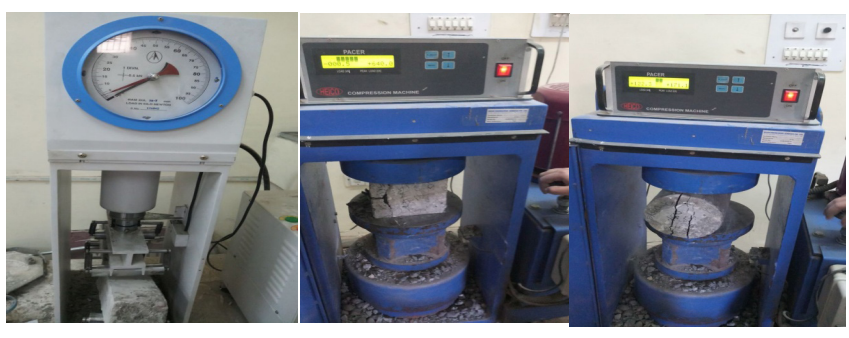

Figure 3. a) Flexural strength b) Compressive strength c) Split tensile strength.

\subsection{Tests on specimens:}

All trials were casting for 7 to 28 days and all trials were de-molded after 24 hours. These are the testing for trials like split tensile test, flexural test and compression test and tested in the universal testing machine.

For each testing three numbers of trials were taken and the result were compared with conventional concrete using average value. These are the tests of failure pattern for split tensile test, flexural test and compression test as shown in Figures $3(\mathrm{a}-\mathrm{c})$, respectively.

\subsection{Details of Density and Strength:}

The density of the PET based concrete for various shape and sizes and corresponding strength has been described in Table 3. Mean Value of strength for 7 and 28 days have been shown in Table 4 .

\section{Results}

\subsection{Compressive Strength Test}

$\mathrm{fc}=\mathrm{P} / \mathrm{A}$

Unit $=\mathrm{N} / \mathrm{mm}^{2}$

$\mathrm{P}=$ failure load in Newton

$\mathrm{A}=$ area subjected compression in $\mathrm{mm}^{2}$

figure, as shown above, optimum strength is obtained at $10 \%$ and also at $20 \%$ with little decrease. However, as the replacement increases beyond $20 \%$, the strength goes on decreasing. Also, after the replacement beyond 30\% is not recommended as samples having replacement of $35 \%$ and $40 \%$ drastically failed. 


\begin{tabular}{|c|c|c|c|c|}
\hline 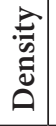 & 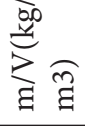 & 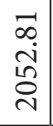 & 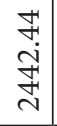 & $\begin{array}{l}8 \\
\stackrel{\sim}{\sim}\end{array}$ \\
\hline $\begin{array}{l}\overrightarrow{7} \\
\overrightarrow{0.00} \\
3 \\
3\end{array}$ & & $\begin{array}{l}\infty \\
\vec{a} \\
\sigma\end{array}$ & $\begin{array}{l}\vec{\sigma} \\
\hat{0}\end{array}$ & $\begin{array}{l}\infty \\
\infty \\
0 \\
0\end{array}$ \\
\hline$\frac{10}{3}$ & ஓे & $\begin{array}{l}\tilde{U} \\
\tilde{J}\end{array}$ & 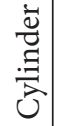 & 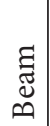 \\
\hline 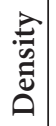 & 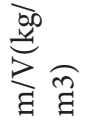 & 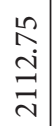 & 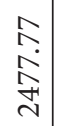 & $\begin{array}{l}0 \\
\infty \\
\vec{\sim}\end{array}$ \\
\hline $\begin{array}{l}\overrightarrow{0} \\
.00 \\
30 \\
3 \\
3\end{array}$ & & $\underset{\sim}{\stackrel{2}{n}}$ & $\stackrel{10}{=}$ & $\begin{array}{l}0 \\
\varrho \\
0\end{array}$ \\
\hline 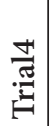 & ㅇํํ & $\begin{array}{l}\tilde{0} \\
\tilde{J}\end{array}$ & 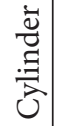 & 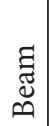 \\
\hline 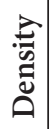 & 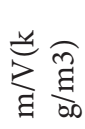 & $\begin{array}{l}0 \\
\infty \\
+ \\
+ \\
0 \\
\vec{N}\end{array}$ & $\begin{array}{l}\hat{\imath} \\
\hat{0} \\
\stackrel{0}{N}\end{array}$ & 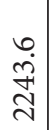 \\
\hline $\begin{array}{l}\overrightarrow{7} \\
.00 \\
3 \\
3\end{array}$ & & $\begin{array}{l}\hat{0} \\
\stackrel{n}{n}\end{array}$ & $\underset{\mathfrak{F}}{\exists}$ & $\begin{array}{l}\infty \\
\stackrel{\infty}{\Xi} \\
=\end{array}$ \\
\hline$\frac{m}{\stackrel{m}{\Xi}}$ & ठ্े & $\begin{array}{l}\tilde{O} \\
\tilde{J}\end{array}$ & $\begin{array}{l}\dot{\vec{v}} \\
\vec{\Xi} \\
\dot{\vec{s}}\end{array}$ & 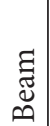 \\
\hline 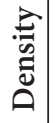 & 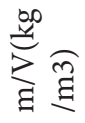 & 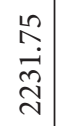 & $\begin{array}{l}= \\
\vec{a} \\
0 \\
\dot{0} \\
\end{array}$ & 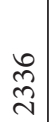 \\
\hline $\begin{array}{l}\overrightarrow{7} \\
.00 \\
30 \\
3\end{array}$ & & $\begin{array}{l}\overrightarrow{\widehat{N}} \\
\stackrel{n}{n}\end{array}$ & $\begin{array}{l}\widetilde{\curvearrowright} \\
\\
=\end{array}$ & $\begin{array}{l}\infty \\
\stackrel{\circ}{\Xi} \\
\exists\end{array}$ \\
\hline 疍 & ڤ્ণ & $\frac{\tilde{J}}{3}$ & 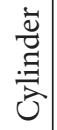 & 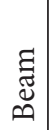 \\
\hline 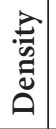 & 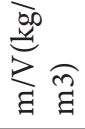 & 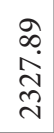 & 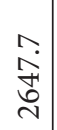 & $\begin{array}{l}\infty \\
\underset{\sim}{\sim} \\
0\end{array}$ \\
\hline $\begin{array}{l}\overrightarrow{0} \\
\frac{5}{50} \\
3 \\
3\end{array}$ & & 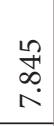 & $\begin{array}{l}10 \\
\vec{a} \\
=\end{array}$ & $\begin{array}{l}\infty \\
\Xi \\
=\end{array}$ \\
\hline $\overrightarrow{.}$ & ¿̊ & Uे & 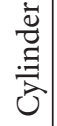 & 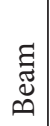 \\
\hline
\end{tabular}

\begin{tabular}{|c|c|c|c|c|c|c|c|c|c|c|c|c|c|c|c|c|c|c|c|}
\hline$\stackrel{\mathbb{E}}{\Sigma}^{\tilde{E}}$ & & \multicolumn{3}{|c|}{ กิ? } & \multicolumn{3}{|c|}{$\stackrel{\infty}{\rightleftarrows}$} & \multicolumn{3}{|c|}{ †े } & \multicolumn{3}{|c|}{$\begin{array}{l}\text { ๙ૂ} \\
\text { فे }\end{array}$} & \multicolumn{3}{|c|}{$\stackrel{n}{0}$} & \multicolumn{3}{|c|}{$\stackrel{\Re}{\stackrel{i}{j}}$} \\
\hline $\begin{array}{l}10 \\
\stackrel{\Xi}{\Xi} \\
\stackrel{\Xi}{\Xi}\end{array}$ & 아 & $\begin{array}{l}\vec{\sim} \\
\stackrel{0}{\sigma}\end{array}$ & $\begin{array}{l}\Delta \\
0 \\
\infty\end{array}$ & 苞 & ت־ & 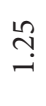 & $\stackrel{\infty}{\rightleftarrows}$ & $\stackrel{\infty}{\stackrel{\Omega}{-}}$ & $\stackrel{2}{2} \stackrel{0}{-}$ & $\stackrel{?}{\rightarrow}$ & $\mid \begin{array}{c}m \\
m \\
\omega \\
n \\
-1\end{array}$ & \begin{tabular}{l}
\multirow{b}{0}{} \\
$\stackrel{-}{-}$
\end{tabular} & $\begin{array}{l}\infty \\
\infty \\
\\
-1\end{array}$ & $\underset{+}{\stackrel{\partial}{+}}$ & $\begin{array}{l}\tilde{b} \\
\dot{r}\end{array}$ & $\stackrel{\substack{n \\
m}}{m}$ & $\begin{array}{l}\stackrel{2}{m} \\
\dot{m}\end{array}$ & $\stackrel{\Rightarrow}{\vec{m}}$ & $\stackrel{\infty}{\stackrel{\infty}{i}}$ \\
\hline 苂 & & & 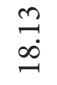 & & & $\stackrel{\overbrace{}}{\stackrel{n}{i}}$ & & & $\stackrel{m}{\stackrel{n}{i}}$ & & & $\begin{array}{l}\stackrel{2}{n} \\
\stackrel{\sim}{n}\end{array}$ & & & $\stackrel{\stackrel{10}{\longrightarrow}}{\underset{+}{+}}$ & & & $\begin{array}{l}n \\
\dot{r} \\
\dot{r}\end{array}$ & \\
\hline$\stackrel{+}{\stackrel{*}{\Xi}}$ & $\stackrel{m}{m}$ & $\begin{array}{c}\hat{n} \\
\end{array}$ & $\mid \begin{array}{c}f \\
+ \\
\infty \\
-1\end{array}$ & $\begin{array}{l}0 \\
\infty \\
\infty \\
\infty \\
-\end{array}$ & $\begin{array}{l}\stackrel{q}{\mathrm{q}} \\
\mathrm{i}\end{array}$ & $\stackrel{\infty}{\vec{i}}$ & $\hat{\widehat{\jmath}}$ & $\stackrel{\sim}{i}$ & $\left.\begin{array}{l}n \\
0 \\
i \\
i\end{array}\right]$ & $\stackrel{\overbrace{}}{i}$ & 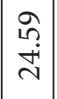 & 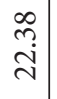 & $\begin{array}{l}\infty \\
\infty \\
\stackrel{i}{i}\end{array}$ & $\stackrel{\infty}{\underset{F}{\not}}$ & $\stackrel{\text { ণे }}{+}$ & $\stackrel{\infty}{\dot{m}}$ & î̀. & $\begin{array}{l}\hat{b} \\
\dot{n}\end{array}$ & $\stackrel{g}{r}$ \\
\hline 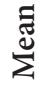 & & & $\begin{array}{l}\text { กุ? } \\
\text { ลे }\end{array}$ & & & $\stackrel{n}{2}$ & & & $\begin{array}{l}\vec{\infty} \\
\vec{i}\end{array}$ & & & $\begin{array}{l}0 \\
\stackrel{-}{\sim}\end{array}$ & & & $\stackrel{\infty}{\stackrel{\infty}{+}}$ & & & $\begin{array}{l}\infty \\
\infty \\
\end{array}$ & \\
\hline $\begin{array}{l}m \\
\stackrel{n}{\Xi} \\
\end{array}$ & in & $\begin{array}{l}\text { ָุ } \\
\end{array}$ & $\mid \begin{array}{l}\infty \\
\stackrel{2}{a} \\
-\end{array}$ & $\vec{i}$ & $\stackrel{n}{\stackrel{m}{m}}$ & $\overrightarrow{\dot{m}}$ & $\begin{array}{l}\infty \\
\stackrel{i}{i}\end{array}$ & $\begin{array}{l}\tilde{O} \\
\dot{r}\end{array}$ & 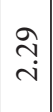 & $\stackrel{\sim}{\vec{m}}$ & $\mid \begin{array}{l}0 \\
0 \\
\dot{0} \\
\vdots\end{array}$ & $\begin{array}{c}\overrightarrow{7} \\
\dot{0} \\
\sim\end{array}$ & 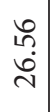 & ถે & $\begin{array}{l}\infty \\
\stackrel{\infty}{+} \\
\stackrel{+}{*}\end{array}$ & $\stackrel{\leftrightarrow}{\rightrightarrows}$ & $\stackrel{f}{\stackrel{+}{*}}$ & $\begin{array}{l}m \\
\infty \\
\dot{m}\end{array}$ & $\underset{i}{\stackrel{N}{i n}}$ \\
\hline
\end{tabular}

\begin{tabular}{|c|c|c|c|c|c|c|c|c|c|c|c|c|c|c|c|c|c|c|}
\hline త్త్రే & & & $\begin{array}{l}\text { fे } \\
\text { \&ें }\end{array}$ & & & $\stackrel{\vec{r}}{\dot{r}}$ & & & $\stackrel{\substack{n \\
i}}{i}$ & & & $\begin{array}{l}\text { זे } \\
\text { ָे }\end{array}$ & & & $\vec{~} \overrightarrow{\text { जे }}$ & & 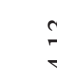 & \\
\hline$\frac{\mathrm{N}}{\stackrel{\Xi}{3}}$ & ¿े & 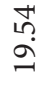 & $\mid \begin{array}{l}\infty \\
\stackrel{\sim}{\sim} \\
\vec{v}\end{array}$ & $\begin{array}{l}\overrightarrow{0} \\
\vec{i}\end{array}$ & $\begin{array}{c}\stackrel{n}{m} \\
i \\
i\end{array}$ & $\begin{array}{c}n \\
\hat{n} \\
n\end{array}$ & $\stackrel{\sim}{m}$ & $\stackrel{\overbrace{}}{i}$ & $\begin{array}{l}\stackrel{\partial}{0} \\
\dot{i}\end{array}$ & 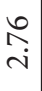 & $\begin{array}{l}0 \\
\stackrel{0}{0} \\
\dot{d}\end{array}$ & $\begin{array}{l}\overrightarrow{0} \\
\dot{i}\end{array}$ & $\begin{array}{c}\overrightarrow{1} \\
\dot{0} \\
\dot{m}\end{array}$ & 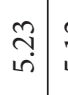 & in & $\begin{array}{l}\infty \\
\substack{++}\end{array}$ & $\begin{array}{l}\overrightarrow{+} \\
+\end{array}$ & 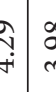 \\
\hline
\end{tabular}

\begin{tabular}{|c|c|c|c|c|c|c|c|c|c|c|c|c|c|c|c|c|c|}
\hline Æే & & $\begin{array}{l}\stackrel{\circ}{\sim} \\
\stackrel{\sim}{\sim}\end{array}$ & & & 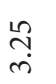 & & & 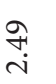 & & & $\begin{array}{l}\text { ro. } \\
\dot{m} \\
\dot{m}\end{array}$ & & $\begin{array}{l}\text { I } \\
\text { in }\end{array}$ & & & 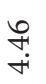 & \\
\hline$\circ$ & iे & 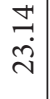 & $\stackrel{\stackrel{\sim}{\sim}}{\stackrel{\sim}{\sim}}$ & $\stackrel{\infty}{\stackrel{\infty}{m}}$ & în & $\stackrel{\infty}{\stackrel{\infty}{i}}$ & 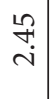 & $\stackrel{\vec{i}}{\vec{i}}$ & \begin{tabular}{l}
$\infty$ \\
\hdashline \\
$i$
\end{tabular} & 芯 & 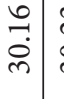 & & \begin{tabular}{l|l}
2 & \\
in & 1 \\
\end{tabular} & $\begin{array}{l}\text { of } \\
\text { in }\end{array}$ & $\stackrel{?}{+}$ & 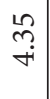 & $\underset{\tau}{\tilde{\tau}}$ \\
\hline
\end{tabular}

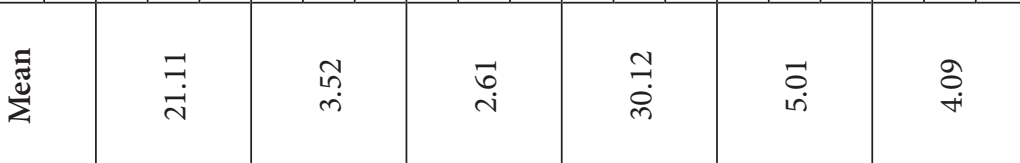

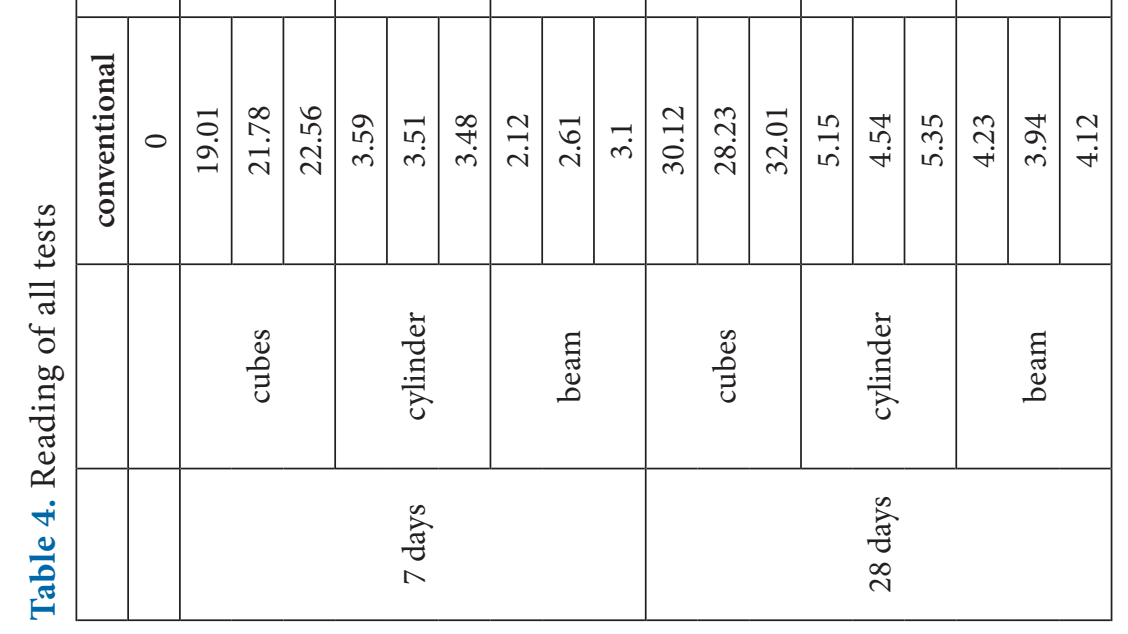




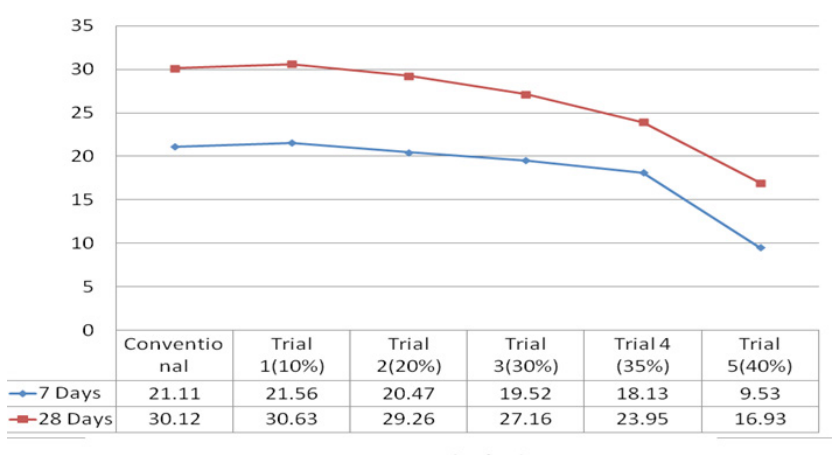

Figure 4. Result of compressive strength.

\subsection{Split-tensile Strength Test}

Suitable formula is as follows:

\section{S.T.S $=2 \mathrm{P} / \pi \mathrm{LD}$}

Where, $\mathrm{P}=$ failure load applied in Newton

$\mathrm{L}=$ specimen's length taken in $\mathrm{mm}$

$\mathrm{d}=$ specimen's width taken in $\mathrm{mm}$

The graph in Figure 5 shows split-tensile-strength of cube with variation in ratio of coarse \& fine aggregate by PET.

For Split tensile test, the replacement of fine and coarse aggregate increase up to $10 \%$ and $20 \%$ shows optimum strength. After 30\% Split tensile strength started to decrease and 35\%, 40\% replacements failed. So we can use the PET aggregate from $10 \%$ to $30 \%$ for the building structure.

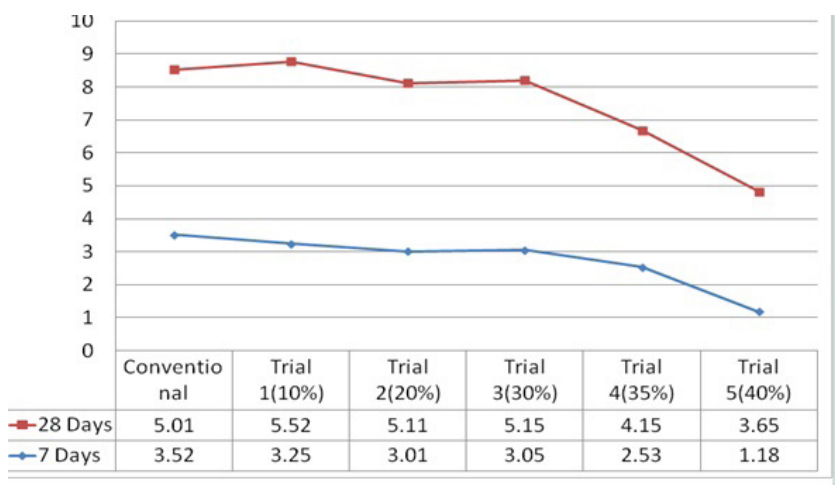

Figure 5. Result of split tensile strength.

\subsection{Flexural Strength Test}

For the flexural strength test the formula is:

Flexural strength test $\mathrm{fb}=\mathrm{Pl} / \mathrm{bd}^{2}$

where, $\mathrm{b}=$ specimen's width in $\mathrm{cm}$.

$\mathrm{d}=$ specimen's depth at point-of-failure.

$1=$ distance to the crack from the support in $\mathrm{cm}$.

$\mathrm{P}=\max$. Load (in $\mathrm{Kg}$ ) acting on the specimen.
The graphic depiction in Figure 6 portrays Flexural strength of cube with varying ratio of coarse \& fine aggregate by PET.

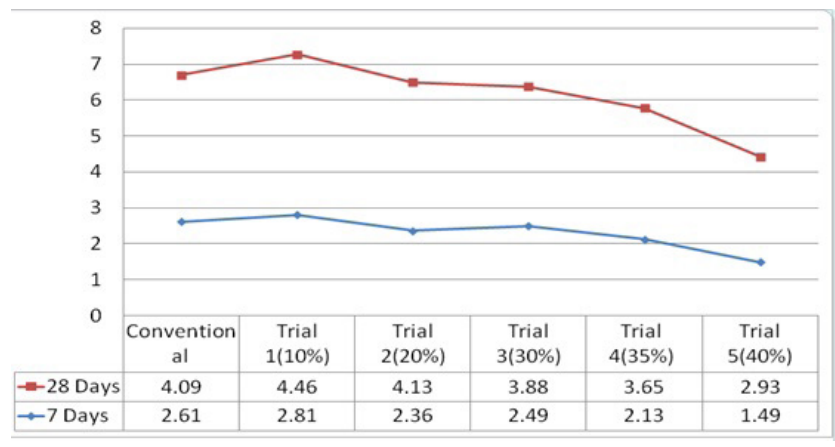

Figure 4. Result of flexural strength.

During testing, the trial samples showed increasing flexural strength up to $10 \%$ of PET. However, on further increment in PET the beam showed decreasing strength. As noted in figure about beyond $10 \%$ of PET is not recommended and beyond $20 \%$ the flexural strength of the beam is unacceptable.

\section{Conclusion}

1. The present paper aimed to find optimum percentage of PET that can be use in construction activities and tried to make concrete light in weight. Also, effect on the density of resulting samples has been observed.

2. Compressive strength trials indicate that PET fine aggregate and coarse aggregate of $10 \%$ and $20 \%$ has comparable strength to conventional concrete cube. It is to be noted that at $10 \%$ of PET the results is a bit better than conventional cube. Beyond 35\% of PET is not recommended.

3. Split tensile test, shows that replacing of fine \& coarse aggregate increases up to $30 \%$ of PET. At $10 \%$, results are higher than conventional concrete. Also, up to $30 \%$ of PET split-tensile strength (cylinder) is comparable to conventional result. Beyond $35 \%$ is no recommended.

4. The Flexural strength trial shows that at $10 \%$ of PET result is most favorable. After $10 \%$ it starts to decrease gradually for $20 \%, 30 \%, 35 \%$ and $40 \%$. More than $35 \%$ is not recommended.

5. Recommended usage for optimum compressive strength is $10 \%$ of PET. Recommended usage for optimum split tensile strength is $10 \%$ or $20 \%$ of PET. 
Recommended usage for optimum flexural strength is $10 \%$ or $20 \%$ of PET. By taking into consideration all the factor and analyzing the obtained experimental values, it is to be concluded that usage of PET as fine as well as coarse aggregate at $10 \%$ and $20 \%$ ratio gives us maximum overall strength.

\section{Discussion}

As we can see from study done till now that PET can be used as a replacement of conventional fine and coarse aggregate. However, the results are not very much improved than conventional and are only of comparable nature. Hence, strength of PET concrete matrix is similar to conventional strength. The main problem faced during the study was to improve the strength of PET concrete matrix as proper bonding is difficult to obtain between. If we are able to solve the problem, then this field has promising future.

The overall strength of PET based concrete at 10\% replacement ratio is good but long term applicable remains an area of concern because no long term study has been done in this regard. Also we don't know how this concrete will behave in different condition of humidity and temperature across different regions of the world. The overall comprehensive strength is dependent on the size of PET aggregate and optimum replacement ratio. The calculations also include type of cement in use and surrounding condition in which concrete setting takes place.

\section{Reference}

1. Saxena AMP, Asolekar SR. Generation of solid-waste in India \& recyclability to be used in building materials. Building and Environment; 2007. p. 2311-20.

2. Thomson J. Environment protection assessment. Report on Plastics. $19^{\text {th }}$ Edition; 2003.

3. Plastic waste in the environment [Internet]. [cited 2017 Jan 24]. Available from: Crossref.

4. Freddy R, Colman E, Rettner B, Treudu P. Europea paradigm study. $3^{\text {rd }}$ Edition. Rostov Books; 2013. PMid:23929756

5. Rashid S, Jainab K, Kaur I. Using recycled plastic in concrete. Waste Management. 2008; 28(10:1835-52. Crossref. PMid:17981022

6. Choilang YW, Mooniker D, Chung JS, Surinder K. Effects of waste PET bottles aggregate on the properties of concrete. Canadian Journal of Civil Engineering. 2005 April; 7(3):1286-91.

7. Cholang YW, Moion DJ, Keim YJ, Lachiemi M. Characterization of mortar-concrete containing fine aggregate manufacturing from recycling waste polyethylene terephthalate bottles. Canadian Journal of Civil Engineering. 2003 May; 4(1):0736-54.

8. Sharma H. Innovative and sustainable application of PET bottle a green construction overview. Indian journal of Science and Technology. 2017; 10(16):1-6. Crossref.

9. Usman N, Masirin MIBM, Ahmad KA, Wurochekke AA, Reinforcement of asphalt concrete mixture using recyclable PET fiber. Indian Journal of Science and Technology. 2016; 9(46).

10. Chelliah AK, Subramaniam MS, Gupta RS, Gupta A. Evaluate the thermo-oxidative degradation of PET using prodegradant additives. Indian Journal of Science and Technology. 2017; 10(6):1-5. Crossref. 